\title{
Control System Design for 3x3 Processes Based on Effective Transfer Function and Fractional Order Filter
}

\author{
G. Hemanth Krishna, R. Kiranmayi, M. Rathaiah
}

\begin{abstract}
Designing of PI/PID controller with fractional order set point filter is proposed to achieve the improved performance of $3 \times 3$ processes. But the design of controller for such higher dimensional multivariable processes is too difficult task because of interaction involved between the process variables. So interaction must be taken into design consideration. In this method of design determines the interaction between the variables using RGA and RNGA methods and uses it in converting the multivariable processes into multiple single loops. The interaction problems between the loops are overcome by incorporating the decouplers in the control loops. Then, the Effective Transfer Function (ETF) has obtained to design PI/PID controller for each individual element as in single input processes. The fractional order filter is also added to improve the servo response of the processes. Hence, the proposed system improves the overall performance by minimizing the interaction effects due to set point variations. This method is also validate by using a case study.
\end{abstract}

Keywords: Interactive process, relative gain array, Effective open loop process, multivariable processes, fractional order filter, decoupler.

\section{INTRODUCTION}

Many of the industrial processes are multi-input and multi-output (MIMO) processes. Tracking of desired performance for these kinds of processes is very intricate compared with single input/output processes because of interactions existing amid the variables [1-3].

The multivariable processes are controlled by two different approaches. These two approaches are popularly known as centralized control and decentralized control schemes. The Decentralized (multi-loop) PI/PID control scheme uses multiple single input single output (SISO) loops. These loops are obtained from multivariable processes based on the strength of interaction existing between the variables.

The motive for using SISO PI/PID controller for Multi-loop control is its trouble-free configuration, straightforward tuning and capability to attain most of the estimated control objectives. It is the common scenario to extend the controller intended technique of SISO systems to multi loop procedures, but it has an effect on the presentation and stability of the systems.
These two approaches are completely depends on the model of the processes. The model identification has been proposed by various researchers in different ways [4]. $\mathrm{Yu}$ and CC [5] have projected the system modeling by means of relay operating in sequential manner. Chidambaram et al [6] have used the relay in feedback for modeling the MIMO systems. Panda and Sujatha [7, 8] discussed about multiple identification methods using relay. Bijeesh et al. [9] used relay for identification of 3 by 3 MIMO process. Shen et al [10] used normalized gain and RGA for modeling the systems.

Xiong et al. [11, 12] and Ho et al. [13] have used independent control design for MIMO systems. Lee et al. [14] have proposed a decentralized control design for MIMO systems by considering interactions. Naik et al. $[15,16]$ proposed a interaction based decentralized control system for interactive processes.

In this, an independent PI controller is designed for specific objective like minimization of interaction amid the control loops and attaining improvement in performance. The interaction among the variables is determined using Relative Gain Array (RGA) and Relative Normalized Gain Array (RNGA) [17]. Then the decentralized controller is designed through the Relative Average Residence Time Array (RARTA) for the pair of manipulated variable (input) and controlled variable (output) suggested by RNGA to achieve the desired performance of the interactive process. To overcome these interaction related problems decoupler have been used in control loops [18, 19]. Even though different decoupler techniques proposed earlier, the dynamic decoupler proposed by Shen et al. [10] is simple and stable. Moreover to achieve the better servo response the filters are used.

The organization of the paper is as follows: Section II discussed the general and proposed structure of MIMO systems. Section III presents interaction measure. Section IV presents the ETF estimation. Section V includes the control system design and Section VI presents the case study and proposed algorithm validation. Also added the conclusions derived from the validation. 


\section{REPRESENTATION OF MULTIVARIABLE PROCESSES}

A schematic representation of general and proposed closed loop control configuration of multivariable processes are shown in Fig.1 and Fig. 2 respectively. In proposed case, the decoupler is used for rejection of loop interaction and set point/ reference filter is for tracking the set point. The elements present in proposed control system are filter $\mathrm{f}_{\mathrm{ii}}(\mathrm{s})$, decentralized controller $\mathrm{G}_{\mathrm{c}}(\mathrm{s})$, decoupler $\mathrm{D}(\mathrm{s})$ and process $\mathrm{G}_{\mathrm{p}}(\mathrm{s})$.

For convenience, it is assumed that the number of manipulated variables in multivariable processes is equal to the number of controlled variables. This allows pairing of single controlled variable with a single manipulated variable through a feedback controller. For pairing of variables, two methods are used namely RGA and RNGA. These two are discussed in section III.

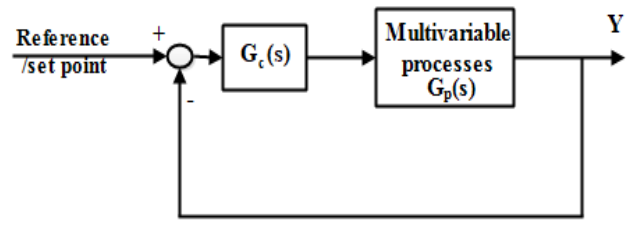

Fig.1.General Structure of closed loop control system

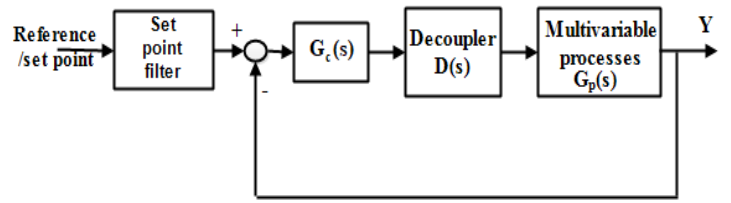

Fig.2.Structure of proposed control system

The conventional representation of the transfer function matrix of the $3 \times 3$ process is:

$$
G_{p}(s)=\left[\begin{array}{lll}
g_{p_{11}}(s) & g_{p 12}(s) & g_{p_{13}}(s) \\
g_{p_{21}}(s) & g_{p_{22}}(s) & g_{p_{23}}(s) \\
g_{p_{31}}(s) & g_{p_{32}}(s) & g_{p_{33}}(s)
\end{array}\right]
$$

Where, the process $\mathrm{g}_{\mathrm{pij}}(\mathrm{s})$ is considered as first order process with delay time (FOPDT), i.e.,

$$
\mathrm{g}_{\text {pij }}(\mathrm{s})=\frac{\mathrm{K}}{\tau \mathrm{s}+1} \mathrm{e}^{-\theta \mathrm{s}}
$$

and the corresponding structure of decentralized controller is of the form,

$$
\mathrm{G}_{\mathrm{c}}(\mathrm{s})=\left[\begin{array}{ccc}
\mathrm{g}_{\mathrm{c} 11}(\mathrm{~s}) & \cdot & \cdot \\
\cdot & \mathrm{g}_{\mathrm{c} 22}(\mathrm{~s}) & \cdot \\
\cdot & \cdot & \mathrm{g}_{\mathrm{c} 33}(\mathrm{~s})
\end{array}\right]
$$

\section{INTERACTION MEASUREMENT}

\section{A. Relative Gain Array(RGA)}

The RGA is defined as follows: Let $K=G_{p}(0)$ be the matrix of steady state gains of the transfer function matrix $G_{p}(s)$, i.e.,

$$
\lim _{s \rightarrow 0} G_{p}(s)=[K]
$$

Further let $\mathrm{R}$ be the transpose of inverse matrix $\mathrm{K}$.

$$
\mathrm{R}=\left[\mathrm{K}^{-1}\right]^{\mathrm{T}}=\left[\mathrm{G}_{p}^{-\mathrm{T}}(0)\right]
$$

Therefore, RGA for $3 \times 3$ systems is,

$$
\Lambda=\mathrm{G}_{\mathrm{p}}(0) \otimes \mathrm{G}_{\mathrm{p}}{ }^{-\mathrm{T}}(0)=\left[\begin{array}{lll}
\lambda_{11} & \lambda_{12} & \lambda_{13} \\
\lambda_{21} & \lambda_{22} & \lambda_{23} \\
\lambda_{31} & \lambda_{32} & \lambda_{33}
\end{array}\right]
$$

Thus, from the equation (6) it is possible to describe the level of interaction, large value of $\lambda_{\mathrm{ij}}$ means that there is strong interaction between corresponding input $i$ and output $j$. If the value of $\lambda_{\mathrm{ij}}$ is greater than 0.5 and approaches towards unity, the interaction also leads between corresponding pairs. In conventional form $\lambda_{\mathrm{ij}}=1$ is represents the ideal case for pairing and avoiding negative pairing.

\section{B. Relative Normalized Gain Array(RNGA)}

By using the two parameters of the process like average residence time (ART) and speed of response, the normalized gain $\left(\mathrm{K}_{\mathrm{N}, \mathrm{ij}}\right)$ of the process can be defined as:

$$
\mathrm{K}_{\mathrm{N}, \mathrm{ij}}=\frac{\mathrm{g}_{\mathrm{ij}}(0)}{\tau_{\mathrm{ar}, \mathrm{ij}}}
$$

This is used for estimating the interaction between the variables of the system. Smaller the $\tau_{\text {ar, }, \mathrm{j}}$ reflects that the large $K_{N, i j}$, similarly, large $\tau_{\text {ar,ij }}$ reflects that the smaller $K_{N, i j}$. In general, $K_{N, i j}$ represents the fastness of the system response in controlled variable with respect to change in manipulated variable. Therefore, the large $K_{N, i j}$ is for all time preferable in control loop pairing. Mathematically, the $\operatorname{ART}\left(\tau_{\mathrm{ar}, \mathrm{ij}}\right)$ can be defined as:

$$
\tau_{a r, i j}=\tau_{i j}+\theta_{i j}
$$

Then the normalized gain is,

$$
\mathrm{K}_{\mathrm{N}, \mathrm{ij}}=\frac{\mathrm{g}_{\mathrm{ij}}(0)}{\tau_{\mathrm{ar}, \mathrm{ij}}}=\frac{\mathrm{k}_{\mathrm{ij}}}{\tau_{\mathrm{ij}}+\theta_{\mathrm{ij}}}
$$

Similarly, $K_{N, i j}$ for $3 \times 3$ process in the form of array is,

$$
K_{N}=\left[\begin{array}{lll}
K_{\mathrm{N}, 11} & K_{\mathrm{N}, 12} & K_{\mathrm{N}, 13} \\
K_{\mathrm{N}, 21} & K_{\mathrm{N}, 22} & K_{\mathrm{N}, 23} \\
K_{\mathrm{N}, 31} & K_{\mathrm{N}, 32} & K_{\mathrm{N}, 33}
\end{array}\right]
$$

It is well-known that the large $K_{N}$ value will become leading loop in process. By the description of relative gain, it is possible to describe the RNGA amid the input and output variables as the relation of normalized gains, i.e.,

$$
\phi_{i j}=\frac{K_{N, i j}}{\hat{K}_{N, i j}}
$$

Where, ' $\hat{K}_{N, i j}$ ' stands for effective gain between input and output variables while all other loops than the current loop is closed. Therefore, the RNGA $(\phi)$ can be expressed as:

$$
\phi=\left[\phi_{i j}\right]_{3 \times 3}=K_{N} \otimes K_{N}^{-T}
$$




\section{EFFECTIVE TRANSFER FUNCTION UISNG RNGA}

To estimate/approximate the equivalent mathematical model of the given process when all loops are open than the current loop to all loops are closed, we first define the relative $\operatorname{ART}\left(\gamma_{\mathrm{ij}}\right)$, i.e.,

$$
\gamma_{i j}=\frac{\hat{\sigma}_{i j}}{\sigma_{i j}}=\frac{\phi_{i j}}{\Lambda_{i j}}
$$

Similarly, representing it is in the array form is called the RARTA which will be expressed as:

$$
\begin{gathered}
\operatorname{RARTA}(\Gamma)=\left[\begin{array}{lll}
\gamma_{11} & \gamma_{12} & \gamma_{13} \\
\gamma_{21} & \gamma_{22} & \gamma_{23} \\
\gamma_{31} & \gamma_{32} & \gamma_{33}
\end{array}\right]=\phi \odot \Lambda \\
\Gamma=\left[\begin{array}{lll}
\phi_{11} & \phi_{12} & \phi_{13} \\
\phi_{21} & \phi_{22} & \phi_{23} \\
\phi_{31} & \phi_{32} & \phi_{33}
\end{array}\right] \odot\left[\begin{array}{lll}
\Lambda_{11} & \Lambda_{12} & \Lambda_{13} \\
\Lambda_{21} & \Lambda_{22} & \Lambda_{23} \\
\Lambda_{31} & \Lambda_{32} & \Lambda_{33}
\end{array}\right]
\end{gathered}
$$

Where, ' $\odot$ ' signifies the Hadamard division/element by element division. From an Eqn. (13), it is simplified as:

$$
\begin{aligned}
& \hat{\sigma}_{i j}=\gamma_{i j} \sigma_{i j} \\
& \hat{\sigma}_{i j}=\hat{\tau}_{i j}+\hat{\theta}_{i j}
\end{aligned}
$$

Using RGA and RARTA, it is likely to write the estimated FOPDT frame as:

$$
\begin{gathered}
\hat{g}_{i j}(s)=\hat{K}_{i j} \frac{1}{\hat{\tau}_{i j} s+1} e^{-\hat{\theta}_{i j} s} \\
\hat{g}_{i j}(s)=\frac{k_{i j}}{\Lambda_{i j}} \frac{1}{\gamma_{i j} \tau_{i j} s+1} e^{-\gamma_{i j} \theta_{i j} s}
\end{gathered}
$$

Then the ETF parameters are identified as:

$$
\begin{gathered}
\hat{K}_{i j}=\frac{k_{i j}}{\Lambda_{i j}} \\
\hat{\tau}_{i j}=\gamma_{i j} \tau_{i j} \\
\hat{\theta}_{i j}=\gamma_{i j} \theta_{i j}
\end{gathered}
$$

\section{THE CONTROL SYSTEM DESIGN}

\section{A. Decoupler Design}

The decoupler is designed by following the concepts of RGA and RNGA control configuration methods. A dynamic decoupler provides better interaction rejection and improved servo performance for sequential changes of set points/reference input. The dynamic decoupler is calculated as:

$$
\begin{aligned}
\Lambda(s) & =\mathrm{G}_{\mathrm{p}}(\mathrm{s}) \otimes \mathrm{G}_{\mathrm{p}}^{-\mathrm{T}}(\mathrm{s}) \\
& =\left[\begin{array}{lll}
\mathrm{g}_{11} & \mathrm{~g}_{12} & \mathrm{~g}_{13} \\
\mathrm{~g}_{21} & \mathrm{~g}_{22} & \mathrm{~g}_{23} \\
\mathrm{~g}_{31} & \mathrm{~g}_{32} & \mathrm{~g}_{33}
\end{array}\right] \otimes\left[\begin{array}{lll}
\mathrm{g}_{11} & \mathrm{~g}_{12} & \mathrm{~g}_{13} \\
\mathrm{~g}_{21} & \mathrm{~g}_{22} & \mathrm{~g}_{23} \\
\mathrm{~g}_{31} & \mathrm{~g}_{32} & \mathrm{~g}_{33}
\end{array}\right]^{-T}
\end{aligned}
$$

\section{B. Fractional Order set point filter}

The fractional order filter is used to enhance the performance of the system under sequential set point changes. The filter is designed as:

$$
f_{i i}(s)=\frac{1+\tau_{t} s}{1+\tau_{c} s^{\alpha+1}}
$$

In this study, the $\tau_{\mathrm{ci}}$ and $\alpha_{i}$ values are selected to attain the similar dynamic performance of the closed loop transfer function for every loop as that of proposed. These parameters are selected as small enough to lessen the outcome of the relations on loop $\mathrm{i}$ and to make sure the stability of the system.

\section{Tuning of controller for ETF of FOPDT dynamics}

The controller being used to attain the expected performance for the system of FOPDT process is given in the form of as given in equation,

$$
g_{c}(s)=k_{c}\left(1+\frac{1}{\tau_{r} s}\right)
$$

The resulting PI controller parameters are given as:

$$
\begin{aligned}
k_{c} & =\frac{k_{o} \tau}{k_{p} \theta} \\
\tau_{r} & =\tau
\end{aligned}
$$

Where, $\mathrm{k}_{\mathrm{o}}$ is chosen as 0.6 to ensure the stability of the system [2].

D.Performance Index

The time integral performance indexes are integral of absolute error (IAE), integral of the square error (ISE). Minimization of IAE and ISE is considered as the objective of present paper.

The time integral performance criteria are expressed as:

$$
\begin{aligned}
& \text { IAE }=\int_{0}^{\infty}\left(\left|e_{1}(t)\right|+\left|e_{2}(t)\right|+\left|e_{3}(t)\right|+\ldots \ldots+\left|e_{n}(t)\right|\right) \\
& \text { ISE }=\int_{0}^{\infty}\left(e_{1}^{2}(t)+e_{2}^{2}(t)+e_{3}^{2}(t)+\ldots \ldots+e_{n}^{2}(t)\right)
\end{aligned}
$$

\section{CASE STUDY \& RESULTS}

Ogunnaike et al [11] have considered the TFM model of OR column, which is being used for design and analysis of proposed method. The process/plant model is given by Eqn. (30).

$$
G_{p}(s)=\left[\begin{array}{lll}
\frac{0.66}{6.7 s+1} e^{-2.6 s} & \frac{-0.61}{8.4 s+1} e^{-3.5 s} & \frac{-0.0049}{9.06 s+1} e^{-6 s} \\
\frac{1.11}{3.25 s+1} e^{-6.5 s} & \frac{-2.36}{5 s+1} e^{-3 s} & \frac{-0.01}{7.09 s+1} e^{-1.2 s} \\
\frac{-34.68}{8.15 s+1} e^{-9.2 s} & \frac{46.2}{10.9 s+1} e^{-9.4 s} & \frac{0.87(11.61 s+1)}{(3.89 s+1)(18.8 s+1)} e^{-s}
\end{array}\right]
$$

This is a three dimensional system. The calculated values of RNGA $(\phi)$ and RARTA $(\Gamma)$ of the OR column are given as:

$$
\Lambda=\left[\begin{array}{ccc}
2.0084 & -0.7220 & -0.2864 \\
-0.6460 & 1.8246 & -0.1786 \\
-0.3624 & -0.1026 & 1.4650
\end{array}\right]
$$




$$
\begin{gathered}
\operatorname{RNGA}(\phi)=\left[\begin{array}{ccc}
1.5504 & -0.4001 & -0.1504 \\
-0.3775 & 1.4557 & -0.0782 \\
-0.1730 & -0.0556 & 1.2286
\end{array}\right] \\
\operatorname{RARTA}(\Gamma)=\left[\begin{array}{lll}
0.7720 & 0.5541 & 0.5251 \\
0.5843 & 0.7978 & 0.4378 \\
0.4773 & 0.5421 & 0.8386
\end{array}\right]
\end{gathered}
$$

The RGA (2.0084), RNGA (1.5504) recommends that the pair of loops 1-1, 2-2, 3-3 for the design of decentralized PI control. The equivalent model parameters and corresponding ETF $\hat{\mathrm{G}}(\mathrm{s})$ is calculated as:

$$
\begin{aligned}
\hat{\mathrm{k}}=\mathrm{G}(0) \odot \Lambda & =\left[\begin{array}{ccc}
0.3286 & 0.8449 & 0.0171 \\
-1.7183 & -1.2935 & 0.0560 \\
95.7001 & -450.3921 & 0.5939
\end{array}\right] \\
\hat{\tau}=\Gamma \otimes \tau & =\left[\begin{array}{lll}
5.1723 & 4.7875 & 4.7570 \\
1.8991 & 3.9891 & 3.1043 \\
3.8897 & 5.9086 & 8.1976
\end{array}\right] \\
\hat{\theta} & =\Gamma \otimes \theta=\left[\begin{array}{lll}
2.0072 & 1.9394 & 0.5251 \\
3.7892 & 2.3934 & 0.5254 \\
4.3908 & 5.0955 & 0.7548
\end{array}\right] \\
\hat{\mathrm{G}}(\mathrm{s}) & =\left[\begin{array}{lll}
0.3286 \\
\frac{0.1723 \mathrm{~s}+1}{\mathrm{e}} \mathrm{e}^{-2.0072 \mathrm{~s}} & \frac{0.8449}{4.7875 \mathrm{~s}+1} \mathrm{e}^{-1.9394 \mathrm{~s}} & \frac{0.0171}{4.7570 \mathrm{~s}+1} \mathrm{e}^{-0.5251 \mathrm{~s}} \\
\frac{-1.7183}{1.8991 \mathrm{~s}+1} \mathrm{e}^{-3.7892 \mathrm{~s}} & \frac{-1.2935}{3.9891 \mathrm{~s}+1} \mathrm{e}^{-2.3934 \mathrm{~s}} & \frac{0.0560}{3.1043 \mathrm{~s}+1} \mathrm{e}^{-0.5254 \mathrm{~s}} \\
\frac{95.7001}{3.8897 \mathrm{~s}+1} \mathrm{e}^{-4.3908 \mathrm{~s}} & \frac{-450.3921}{5.9086 \mathrm{~s}+1} \mathrm{e}^{-5.0955 \mathrm{~s}} & \frac{0.5939}{8.1976 \mathrm{~s}+1} \mathrm{e}^{-0.7548 \mathrm{~s}}
\end{array}\right]
\end{aligned}
$$

The ETF considered for design of PI controller is given as: $\hat{\mathrm{G}}(\mathrm{s})=\operatorname{diag}\left\{\frac{0.3286}{5.1723 \mathrm{~s}+1} \mathrm{e}^{-2.0072 \mathrm{~s}}, \frac{-1.2935}{3.9891 \mathrm{~s}+1} \mathrm{e}^{-2.3934 \mathrm{~s}}, \frac{0.5939}{8.1976 \mathrm{~s}+1} \mathrm{e}^{-0.7548 \mathrm{~s}}\right\}$

For comparing the effectiveness of the proposed method, the PI controller parameters are also calculated using SIMC method [4] and Ziegler-Nicholas tuning formulas. The calculated controller setting for these methods are listed in Table 1.

\begin{tabular}{|c|c|c|c|c|c|c|}
\hline \multirow{2}{*}{$\begin{array}{l}\text { Control } \\
\text { loop }\end{array}$} & \multicolumn{2}{|c|}{ Proposed } & \multicolumn{2}{|c|}{ SIMC PI [4] } & \multicolumn{2}{|c|}{$\begin{array}{l}\text { Ziegler-Nichols } \\
\text { PI }\end{array}$} \\
\hline & $k_{c}$ & $\tau_{i}$ & $k_{c}$ & $\tau_{i}$ & $k_{c}$ & $\tau_{i}$ \\
\hline $\mathrm{y}_{1}-\mathrm{u}_{1}$ & 1.994 & 4.35 & 1.383 & 3.535 & 1.08 & 4.25 \\
\hline $\mathrm{y}_{2}-\mathrm{u}_{2}$ & -0.422 & 4.295 & -0.148 & 1.666 & -0.233 & 3.32 \\
\hline $\mathrm{y}_{3}-\mathrm{u}_{3}$ & 2.825 & 4.191 & 5.0 & 9.979 & 2.78 & 5.24 \\
\hline $\begin{array}{r}\frac{1.183}{64.5} \\
\frac{-52.45}{4.5}\end{array}$ & $\begin{array}{l}3.164 \\
\frac{95 s+1)}{5(5+1)} e^{-0 .} \\
\frac{513 s+1)}{5 s+1)} e^{-1}\end{array}$ & \multicolumn{3}{|c|}{$s \frac{-0.581(1.355 \mathrm{~s}+1)}{(6.22 \mathrm{~s}+1)} \mathrm{e}^{-0.365 \mathrm{~s}}$} & \multicolumn{2}{|c|}{$\begin{array}{c}\frac{0.010(25 \mathrm{~s}+1)}{(5.745 \mathrm{~s}+1)} \mathrm{e}^{-2.008 \mathrm{~s}} \\
\frac{-0.002814(9.88 \mathrm{~s}+1)}{(5.745 \mathrm{~s}+1)} \mathrm{e}^{-7.725 \mathrm{~s}} \\
1.666\end{array}$} \\
\hline
\end{tabular}

The decoupler $\mathrm{D}(\mathrm{s})$ is also calculated and given in eqn. (31).

Table 1:

SIMC controller parameters for Different approaches for OR column

(31)

The set point filters for all three loops are calculated as:

$$
\begin{aligned}
& \mathrm{f}_{11}(\mathrm{~s})=\frac{1}{1+9 \mathrm{~s}^{1.06}}(4 \mathrm{~s}+1) \\
& \mathrm{f}_{22}(\mathrm{~s})=\frac{1}{1+6 \mathrm{~s}^{1.08}}(2 \mathrm{~s}+1)
\end{aligned}
$$

$$
\mathrm{f}_{33}(\mathrm{~s})=\frac{1}{1+0.4 \mathrm{~s}^{1.06}}(0.1 \mathrm{~s}+1)
$$

The filters are calculated for the frequency range of $10^{-3}$ to $10^{3}$.

The simulation results of first, second and third output of OR column process is shown in Fig.3, Fig.4 and Fig. 5 respectively. The performance indices are shown in Table 2.

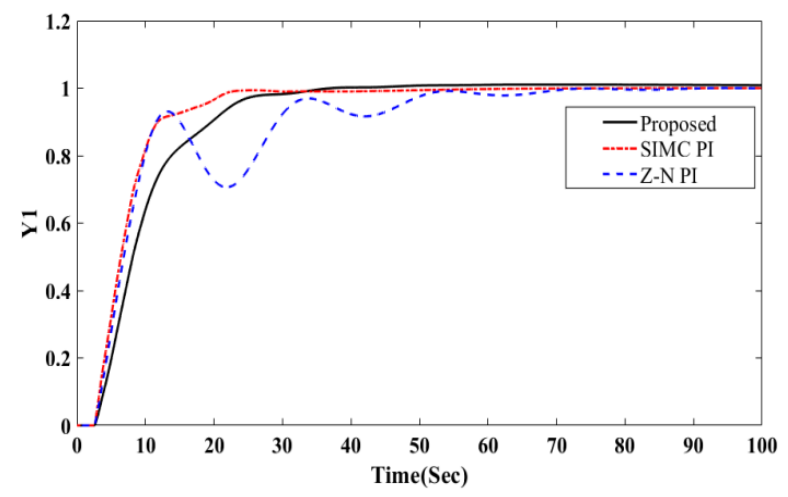

Fig.3.Closed loop response of first output when $r_{1}=1$ and $r_{2}=r_{3}=0$

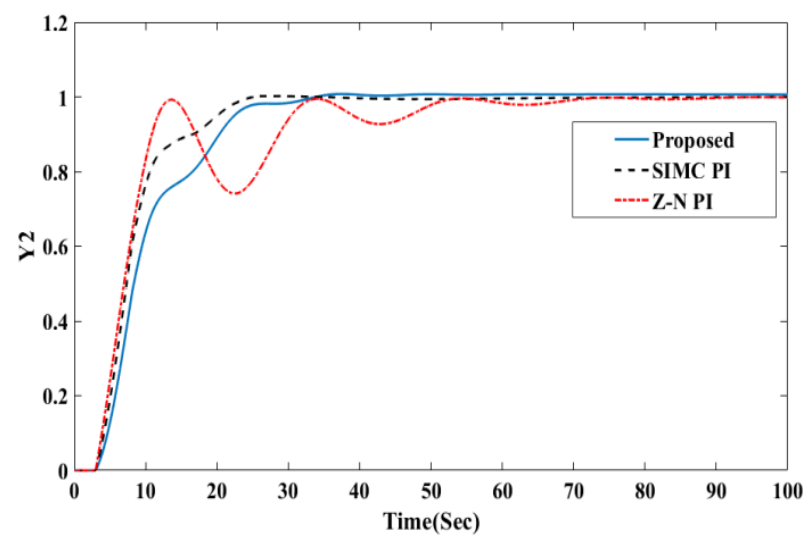

Fig.4. Closed loop response of second output when $r_{2}=1$ and $r_{1}=r_{3}=0$

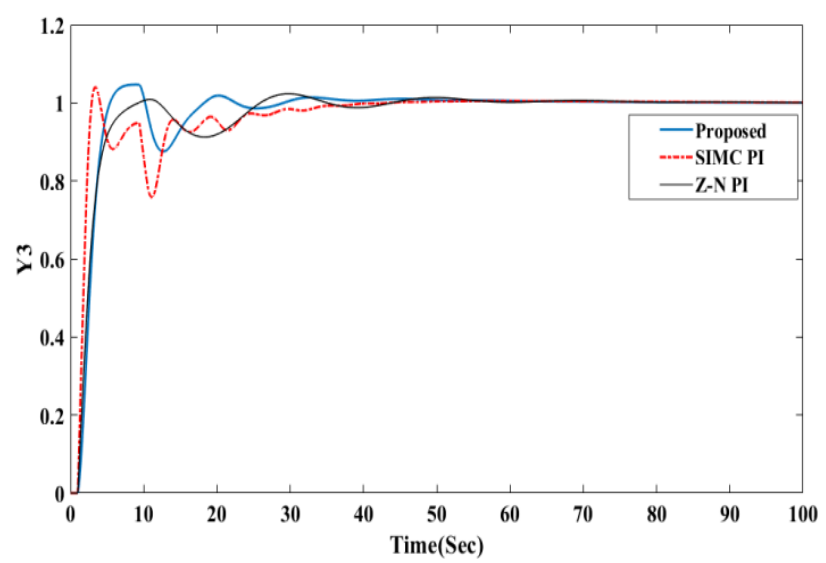

Fig.5. Closed loop response of third output when $r_{3}=1$ and $r_{1}=r_{2}=0$ 
Table 2:

Performance indices of $\mathrm{OR}$ column

\begin{tabular}{|c|c|c|c|}
\hline \multirow{2}{*}{$\begin{array}{c}\text { Reference/ } \\
\text { set point }\end{array}$} & $\begin{array}{c}\text { Control } \\
\text { Method }\end{array}$ & $\begin{array}{c}\text { IAE } \\
\text { (Sum of } \\
\text { IAE) }\end{array}$ & $\begin{array}{c}\mathbf{I S E}_{\mathbf{t}} \\
\text { (Sum of } \\
\text { ISE) }\end{array}$ \\
\hline \multirow{3}{*}{$\begin{array}{c}\mathrm{r}_{1}=1 \\
\left(\mathrm{r}_{2}=\mathrm{r}_{3}=0\right)\end{array}$} & Proposed & 53.67 & 46.219 \\
\cline { 2 - 4 } & SIMC PI & 57.609 & 57.085 \\
\cline { 2 - 4 } & Z-N PI & 208.78 & 1482.316 \\
\cline { 2 - 4 } $\mathrm{r}_{2}=1$ \\
$\left(\mathrm{r}_{1}=\mathrm{r}_{3}=0\right)$ & Proposed & 14.6051 & 4.01935 \\
\hline \multirow{3}{*}{$\begin{array}{c}\mathrm{r}_{3}=1 \\
\left(\mathrm{r}_{1}=\mathrm{r}_{2}=0\right)\end{array}$} & SIMC PI & 15.6798 & 7.148576 \\
\cline { 2 - 4 } & Z-N PI & 57.034 & 100.3601 \\
\cline { 2 - 4 } & Proposed & 3.74861 & 1.80 \\
\hline
\end{tabular}

From the results obtained, it is clear that the proposed method has achieved the expected reference/set point as quickly as possible than the methods considred for comparison. The response of first output $\left(\mathrm{Y}_{1}\right)$ when $\mathrm{r}_{1}=1$ is shown in Fig. 3 and the corresponding IAE, ISE values are 53.67 and 46.219 respectively. Lesser the IAE and ISE better the performance. It is smaller value than the methods SIMC

Similarly, the response of second output $Y_{2}$ when $r_{2}=1$ is shown in Fig. 4 and the corresponding IAE, ISE values are 14.6051 and 4.01935 respectively. This value is also shows the improved response of than the other two methods.

The response of the third output $Y_{3}$ when $r_{3}=1$ is shown in Fig.5 and the corresponding IAE, ISE values are 3.74861 and 1.80 respectively. This value indicates that proposed method has improved the performance of the system than the SIMC PI and ZN PI approaches. The performance has improved only due to the presence of decoupler and filter in the design.

\section{CONCLUSIONS}

In this paper, a decentralized controller is projected for Interactive multi-time delay processes. First, an interaction among the variables is obtained by the RGA method. But this method considers only the steady state gains of the processes. So to include the dynamics in measurement the normalized gain of process elements has been calculated. According to the RGA and RNGA, the pairing is formed and these are diagonalised in square processes. From the relations of RGA and RNGA, an effective transfer function has been determined. Then the PI controller is tuned using the algorithm. To overcome the interaction, decoupler has been included in the loop. The performance of the proposed controller is investigated through applying it to three different processes with (first order process with multi time delay) FOPMDT. To show the effectiveness of the proposed PI controller, the simulation results and different time integral performance are compared with relevant tuning techniques. With this proposed method the objectives has been achieved in a better way than the conventional approaches PI and ZN PI (from Table 2).

\section{REFERENCES}

1. Q. Xiong, W.-J. Cai, M.-J. He, A practical loop pairing criterion for multivariable processes, Journal of Process Control 15 (2005) 741-747.

2. H.-P. Huang, J.-C. Jeng, C.-H. Chiang, W. Pan, A direct method for multi-loop PI/PID controller design, Journal of Process Control 13 (2003) 769-786.

3. Naik, R. Hanuma, DV Ashok Kumar, and K. S. R. Anjaneyulu. "Loop Interaction and It's Influence in Multivariable Process Control." Emerging Trends in Electrical, Communications and Information Technologies. Springer, Singapore, 2017. 361-368.

4. Kishore, D., Smruti Rajan, S., Anand Kishore, K., \& Panda, R. C. (2019). An improved identification and control of $3 \times 3$ multi-input multi-output system using relay and subspace method. Indian Chemical Engineer, 61(1), 87-101.

5. Yu, Cheng-Ching. Autotuning of PID controllers: A relay feedback approach. Springer Science \& Business Media, 2006.

6. Chidambaram M and Padma Sree, Control of unstable and single and multi variable systems, Narosa Publications, New Delhi , 2017.

7. Panda, Rames C., and V. Sujatha. "Identification and Control of Multivariable Systems-Role of Relay Feedback." Introduction to PID Controllers-Theory, Tuning and Application to Frontier Areas. IntechOpen, 2012.

8. Panda, Rames C. Introduction to PID Controllers-Theory, Tuning and Application to Frontier Areas. 2012.

9. Bijeesh, K., D. Kalpana, and T. Thyagarajan "Identification of 3-by-3 MIMO system using relay feedback." 2014 IEEE International Conference on Advanced Communications, Control and Computing Technologies. IEEE, 2014.

10. Shen, Yuling, Wen-Jian Cai, and Shaoyuan Li. "Normalized decoupling control for high-dimensional MIMO processes for application in room temperature control HVAC systems." Control Engineering Practice 18.6 (2010): 652-664.

11. Qiang xiong,Wen-Jian Cai, Mao-Jun He, Equivalent transfer function method for PI/PID controller design of MIMO processes, J.Process control 17(2007) 665-673.

12. Q. Xiong, W.-J. Cai, Effective transfer function method for decentralized control system design of multi-input multi-output processes, Journal of Process Control 16 (2006) 773-784.

13. W. H. Ho, T. H Lee, and O. P. Gan, "Tuning of multi-loop PID controllers based on gain and phase margin specifications," Ind. Eng. Chem. Res., vol. 36, pp. 2231-2238, 1997.

14. Truong Nguyen Luan $\mathrm{Vu}$ and Moonyong Lee,"Independent design of Multiloop PI/PID controllers for Multi-delay processes", world academy science, Engineering and technology,(60)2009,PP 703-708.

15. Naik, R. Hanuma, D. V. Kumar, and K. S. R. Anjaneyulu. "Controller for multivariable processes based on interaction approach." Int J Appl Eng Res 7.2012 (2012): 1203-1213.

16. Naik, R. Hanuma, DV Ashok Kumar, and K. S. R. Anjaneyulu. "Control configuration selection and controller design for multivariable processes using normalized gain." World Acad Sci Eng Technol Int J Electr Comput Electron Commun Eng8.10 (2014).

17. He, Mao-Jun, et al. "RNGA based control system configuration for multivariable processes." Journal of Process Control 19.6 (2009): 1036-1042.

18. Rajapandiyan, C., and M. Chidambaram. "Controller design for 
MIMO processes based on simple decoupled equivalent transfer functions and simplified decoupler." Industrial \& Engineering Chemistry Research 51.38 (2012): 12398-12410.

19. Naik, R. Hanuma, DV Ashok Kumar, and K. S. R. Anjaneyulu. "A Comparative Study of Decoupler Design Techniques for TITO Control Processes." Emerging Trends in Electrical, Communications and Information Technologies. Springer, Singapore, 2017. 293-301. 